ISSN 2447-9071

doi https://doi.org/10.36414/rbmc.v6i16.61

Contato para correspondência:

Rogério José de Almeida

E-mail:

rogerio.almeida@faculdadepm.edu.br

Conflito de interesse: Não

Financiamento: Recursos próprios

Recebido: 09/11/2020

Aprovado: 30/11/2020

\section{Fatores associados aos níveis de estresse percebido em estudantes internos de um curso de medicina}

\section{Factors associated with levels of perceived stress in internal students of a medicine course}

Jordana Nascimento Machado', Luciana Benevides de Araújo', Érika Guimarães Nogueira', Nathália Camargo de Matos' ${ }^{1}$ Antonio Márcio Teodoro Cordeiro Silva 1,2, Rogério José de Almeida ${ }^{1,2}$

${ }^{1}$ Pontifícia Universidade Católica de Goiás - PUC Goiás

${ }^{2}$ Faculdade da Polícia Militar - FPM

\begin{abstract}
Resumo
Aformaçãomédica demandaatençãoporparte da gestão das escolas médicas. Oestágio do internato, fase final do curso de medicina, é decisivo nesta formação. Assim, o objetivo deste estudo foi analisar os fatores associados a maiores níveis de estresse percebido em estudantes interno de um curso de medicina. Trata-se de um estudo observacional, de corte transversal e analítico. Dois questionários foram aplicados, sendo um com dados sociodemográficos eoutro com a Escala de Estresse Percebido, que mensura o grau no qual os indivíduos percebem as situações como estressantes. Foram incluídos napesquisa 140 acadêmicos do internato médico, sendo $67,9 \%$ com idade até2 24 anose $70,7 \%$ do sexo feminino. Amaioria era solteiro (54,2\%), procedentes de Goiânia (53,6\%), moravam comos pais (68,3\%) e faziam terapia psiquiátrica/psicológica (60,6\%). Na comparação dos aspectos sociodemográficos com os níveis de estresse percebido dos estudantes foi identificado maior escore no sexo feminino ( $p$ $=0,0053)$, bem como nos alunos que referiram não realizar atividades extracurriculares $(p=0,0105)$. Em relaçãoà comparação dos aspectos pessoais e clínicos com os níveis deestresse percebido, maiores escores foram encontrados em quem referiu ter doença psiquiátrica diagnosticada ( $p=0,0273)$, nos que estavam fazendo terapia psiquiátrica ou psicológica $(p=0,0003)$, nos que refeririam ter insônia $(p=0,0099)$, nos que dormiam menos de sete horas por noite $(p=0,0161)$, nos estudantes que afirmaram não estar satisfeitos com o rendimento acadêmico $(p<0,0001)$ e naqueles que já pensaram em abandonar o curso de medicina $(p=0,0016)$. Evidenciaram-se fatores associados aos níveis de estresse desses estudantes nos dois últimos anos do curso de medicina, tais como: sexo feminino, não fazer atividade extracurricular, ter doenças psiquiátricas, frequentar terapia psiquiátrica/psicológica, quantidade de horas dormidas ànoite reduzida, insônia, insatisfação com o rendimento acadêmicoe pensamento deabandonaro curso. Esteestudo, embora tenha sido restrito ao internato, viabiliza dados que merecem atenção das escolas médicas na perspectiva de melhorar a vida diária dos discentes.
\end{abstract}

Palavras-Chave: Estudantes de Medicina, Estresse Percebido, Internato Médico, Saúde Mental.

\begin{abstract}
Medical training demands attention from the management of medical schools. The medical internship, the final phase of the medical course, is decisive in this training. Thus, the aim of this study was to analyze the factors associated with higher levels of perceived stress in medical school students. This is an observational, cross-sectional and analytical study. Two questionnaires were applied, one with sociodemographic data and the other with the Perceived Stress Scale, which measures the degree to which individuals perceive situations as stressful. 140 students from the medical internship were included in the research, $67.9 \%$ aged up to 24 years and $70.7 \%$ female. Most were single (54.2\%), from Goiânia (53.6\%), lived with their parents (68.3\%) and underwent psychiatric / psychological therapy
\end{abstract}


(60.6\%). perceived stress levels of students, a higher score was identified in females ( $p=0.0053)$, as well as in students who reported not performing extracurricular activities $(p=0.0105)$, in relation to the comparison of personal and clinical aspects with perceived stress levels, higher scores were found in those who reported having diagnosed psychiatric illness $(p=0.0273)$, in those who were undergoing psychiatric or $p$ sychological therapy $(p=0.0003)$, in those who reported having insomnia ( $p=0$, 0099), in those who slept less than seven hours a night $(p=0.0161)$, in students who said they were not satisfied with academic performance $(p<0.0001)$ and in those who had already thought about leaving the medical school ( $p=0.0016$ ). Factors associated with stress levels students in the last two years of medical school, such as: female gender, not do extracurricular activities, having psychiatric illnesses, being attending psychiatric/psychological therapy, reduced number of hours spent at night, insomnia, dissatisfaction with academic performance and thought of abandoning the course. This study, although it was restricted to boarding school, provides data that deserve attention from medical schools in order to improve students' daily lives.

Keywords: Medical students, Medical internship, Perceived stress, Mental health.

\section{Introdução}

Conceitualmente, pode-se assumir o estresse como uma ameaça verdadeira ou fictícia ao estado de harmonia do organismo humano que tem a competência de interferir nas respostas mais apropriadas e previstas em determinada situação, sendo apontado como um fator deletério à saúde e ao bemestar das pessoas. Apontado como um dos principais males que afetam as pessoas hodiernamente, o estresse influencia o homem em suas mais variadas maneiras de convívio social'.

Diferentes situações e fatores são considerados estressantes na formação em medicina. Alguns fatores são: método de estudo, provas e testes, manejo do tempo, conflitos entre dever e prazer, constante disputa entre os estudantes, vivências relacionadas com a morte, intimidade corporal e emocional no exame com o paciente, apreensão em adquirir doenças, impasses éticos, evidências de que o médico não é onipotente, aflições quanto à retenção das informações ao longo do curso, preocupação com os lucros financeiros vindouros, plantões do internato, decisão acerca da especialidade e o sofrimento pelo medo de não ser aprovado no exame de residência médica².

Ao iniciar o curso de medicina, o estudante precisa se adaptar a uma realidade nova de aquisição e repasse do conhecimento, da vida na faculdade, de modificação no estilo de vida e de obtenção de maiores incumbências, para as quais talvez ainda não esteja pronto. $O$ contato com doentes críticos, com o sofrimento e com a morte também pode simbolizar significativa fonte de estresse ao aluno já nos estágios iniciais do curso, gerando uma enorme aflição diante de um protótipo tradicional, com perspectiva científica e não emocional por parte das instituições médicas, que não vislumbram alinhar seus alunos para tais ocorrências'.
Parte componente da graduação, o estágio curricular essencial de treinamento em serviço, ocorre nos dois últimos anos da graduação e é feito em sistema de internato. Realizado em serviços próprios ou conveniados, acontece sob vigilância dos professores da determinada escola em questão. A carga horária mínima do estágio curricular deve atingir 2.700 horas, um total de $35 \%$ da carga horária total mínima do curso, que é de 7.200 horas $^{2}$.

É no internato que a dedicação integral faz com que vários alunos reconsiderem sua decisão profissional, gerando angústia e causando estresse. Nos estudantes de medicina, o estresse é particularmente preocupante, porque ao afetar funções fisiológicas, psicológicas e cognitivas, danifica também sua qualidade de vida e intervém no aprendizado e no cuidado ao paciente. Assim, o desmazelo do bem-estar desses acadêmicos, além de uma questão de saúde individual, constitui uma questão de saúde pública, uma vez que, ao danificar a instrução dos futuros médicos, produzirá malefícios que recairão também sobre os usuários ${ }^{4}$.

Nesse contexto, pode-se inferir que a cultura médica divulga e aceita a ideia de que alunos de medicina e médicos não conseguem ter um estilo de vida saudável. Inseridos nessa conjuntura, a ideia do médico como super-herói continua sendo propagada, e eles aprendem a descuidar das próprias necessidades, o que os deixa mais expostos a sintomas psicológicos e ao uso de drogas, arruinando a construção da sua identidade profissional, como também o zelo que terão com o paciente ${ }^{5}$.

Nesse sentido, a análise da problematização empreendida situa-se na busca de variáveis que podem estar relacionadas ao estresse percebido em estudantes internos de medicina. Assim, este estudo teve por objetivo analisar os fatores asso- 
ciados a maiores níveis de estresse percebido em estudantes interno de um curso de medicina.

\section{Métodos}

Trata-se de um estudo observacional, de corte transversal e analítico. Este é um método de pesquisa que visualiza a situação de uma população em um determinado momento, como instantâneos da realidade. Identificados dentro de uma população os desfechos existentes, pode-se levantar fatores que podem ou não estar associados a esses resultados em diferentes níveis de associação 6 .

A pesquisa foi realizada por meio de questionários aplicados aos estudantes internos do curso de Medicina da Pontifícia Universidade Católica de Goiás (PUC Goiás) no período de janeiro a fevereiro de 2020. Como critérios de inclusão foram selecionados estudantes que estavam cursando do nono ao décimo segundo período e que estavam regularmente matriculados. Foram excluídos da pesquisa os estudantes em licença médica, que não estavam frequentando regularmente o internato e que não responderam a todos os itens dos questionários.

Dois instrumentos de pesquisa autoaplicáveis foram utilizados neste estudo. O primeiro foi um questionário sociodemográfico, pessoal e clínico construídos pelos pesquisadores cujo objetivo foi levantar dados dos estudantes que possam ter associação com os níveis de estresse percebido.

O segundo questionário foi a Escala de Estresse Percebido, que mensura o grau no qual os indivíduos percebem as situações como estressantes. Esta escala, denominada Perceived Stress Scale (PSS) foi traduzida para o português brasileiro adaptada e validada para estudantes universitárias ${ }^{7}$. O objetivo é avaliar o nível em que um indivíduo aprecia as suas circunstâncias de vida como estressantes. A escala original inclui 14 itens que são questões relativas ao sentimento ou pensamento do indivíduo no mês anterior ao momento da pesquisa ${ }^{7}$.

A PSS 14 se constitui em uma escala unidimensional cuja nota global resulta da soma dos valores atribuídos a cada item. As respostas são dadas em uma escala tipo Likert de cinco posições (que recebem uma classificação entre 0 e 4). Metade dos itens são formulados de forma positiva, que são designados na literatura como itens positivos, e metade negativa, onde, para chegar à nota total os valores dos itens devem ser somados e revertidos pela positiva, de forma que a uma nota mais elevada corresponda maior estresse ${ }^{7}$.

A PSS resulta em uma pontuação que varia de 0 a 56. A soma dos escores referentes à cada sentença é utilizada para calcular o escore geral de estresse percebido, sendo que quanto maior o escore, mais alto é o nível de estresse percebido7.
Após a coleta dos resultados, foi confeccionado um banco de dados utilizando o software IBM SPSS Statistics 18. Em seguida, foi realizada estatística descritiva com o cálculo medidas de tendência central para as variáveis contínuas e cálculo das frequências absoluta e relativa percentual para as variáveis discretas.

Na sequência foi aplicado teste de normalidade (Kolmogorov-Smirnov) para distinguir as distribuições paramétricas e não-paramétricas, com o intuito de comparação dos resultados do questionário estratificado pelas variáveis sociodemográficas. Foram utilizados, para as distribuições paramétricas, os testes $t$ de Student e ANOVA e para as distribuições não-paramétricas os testes Mann-Whitney e KruskalWallis. Para todos os testes comparativos foi assumido $p$-valor menor ou igual a 0,05 como significativo.

Antes de iniciar a coleta de dados, o presente trabalho foi encaminhado ao Comitê de Ética e Pesquisa (CEP) da Pontifícia Universidade Católica de Goiás (PUC Goiás), onde foi aprovado com o parecer n. 3.425.379. Todos os participantes foram informados dos objetivos da pesquisa e assinaram o Termo de Consentimento Livre e Esclarecido.

\section{Resultados}

Os dados sociodemográficos dos 140 acadêmicos internos entrevistados demonstraram que $70,7 \%$ eram do sexo feminino e $29,3 \%$ masculino. Com relação à idade, $67,9 \%$ dos participantes tinham até 24 anos, com uma média de $24,3( \pm 3,4)$ e idade mínima de 21 e máxima de 49 anos. Um total de $54,2 \%$ eram solteiros e $53,6 \%$ procedentes de Goiânia. Observou-se que $36,6 \%$ dos acadêmicos trabalham em concomitância com o curso de Medicina e que $60,6 \%$ realizavam pelo menos uma atividade extracurricular. A maioria dos estudantes (68,3\%) morava com os pais e $26,1 \%$ moravam sozinhos (Tabela 1 ).

Tabela 1. Caracterização dos aspectos sociodemográficos dos 140 estudantes internos de um curso de medicina, Goiânia, Goiás, Brasil, 2020.

\begin{tabular}{lcc}
\hline Variáveis $(\mathbf{N}=\mathbf{1 4 0})$ & $\boldsymbol{n}$ & $\boldsymbol{f}(\%)$ \\
\hline Sexo & 41 & 29,3 \\
Masculino & 99 & 70,7 \\
Feminino & & \\
Idade (anos) & 95 & 67,9 \\
Até 24 anos & 45 & 32,1 \\
Acima de 24 anos & & \\
Procedência & 80 & 56,3 \\
Goiânia & 36 & 25,4 \\
Interior de Goiás & 24 & 16,9 \\
Outro Estado & &
\end{tabular}


Trabalha e cursa medicina

\begin{tabular}{|c|c|c|}
\hline Sim & 52 & 36,6 \\
\hline Não & 88 & 62,0 \\
\hline \multicolumn{3}{|l|}{ Período de curso } \\
\hline Período 9 & 27 & 19,0 \\
\hline Período 10 & 31 & 21,8 \\
\hline Período 11 & 37 & 26,1 \\
\hline Período 12 & 45 & 31,7 \\
\hline \multicolumn{3}{|c|}{ Atividades Extracurriculares } \\
\hline Nenhuma & 9 & 6,3 \\
\hline 1 Atividade & 86 & 60,6 \\
\hline 2 Atividades & 38 & 26,8 \\
\hline 3 ou mais Atividades & 7 & 4,9 \\
\hline \multicolumn{3}{|l|}{ Estado Civil } \\
\hline Solteiro & 77 & 54,2 \\
\hline Solteiro (Namorando) & 59 & 41,5 \\
\hline Casado & 4 & 2,8 \\
\hline \multicolumn{3}{|l|}{ Envolvimento Religioso } \\
\hline Forte & 44 & 31,0 \\
\hline Fraco & 67 & 47,2 \\
\hline Nenhum & 29 & 20,4 \\
\hline \multicolumn{3}{|l|}{ Mora com quem? } \\
\hline Pai, mãe ou irmãos & 97 & 68,3 \\
\hline Sozinho & 37 & 26,1 \\
\hline Parentes & 6 & 4,2 \\
\hline
\end{tabular}

A caracterização dos aspectos pessoais e clínicos evidenciou que metade dos estudantes praticava atividade física frequentemente. Além disso 62,0\% negaram possuir alguma doença psiquiátrica; no entanto, $60,6 \%$ disseram frequentar terapia psiquiátrica/psicológica. Grande parte dos acadêmicos $(81,0 \%)$ dorme menos de 7 horas por noite e $58,5 \%$ relataram fazer uso de substâncias que alteram a qualidade do sono. Apesar de $68,3 \%$ dos estudantes relatarem insatisfação com o rendimento acadêmico, menos da metade já pensou em abandonar o curso (Tabela 2).

Tabela 2. Caracterização dos aspectos pessoais e clínicos dos 140 estudantes internos de um curso de medicina, Goiânia, Goiás, Brasil, 2020.

\begin{tabular}{lll}
\hline Variáveis (N=140) & $\boldsymbol{n}$ & $\boldsymbol{f ( \% )}$ \\
\hline Frequência em Bares e Festas & & \\
Frequentemente & 49 & 34,5 \\
Às vezes & 65 & 45,8 \\
Raramente & 26 & 18,3 \\
Frequência Atividade Física & & \\
Frequentemente & 71 & 50,0 \\
Às vezes & 37 & 26,1 \\
Raramente & 32 & 22,5
\end{tabular}

\begin{tabular}{|c|c|c|}
\hline \multicolumn{3}{|l|}{ Doença Crônica } \\
\hline Sim & 12 & 8,5 \\
\hline Não & 128 & 90,1 \\
\hline \multicolumn{3}{|l|}{ Doença Psiquiátrica } \\
\hline Sim & 52 & 36,6 \\
\hline Não & 88 & 62,0 \\
\hline \multicolumn{3}{|c|}{ Terapia Psiquiátrica/Psicológica } \\
\hline Sim & 86 & 60,6 \\
\hline Não & 54 & 38,0 \\
\hline \multicolumn{3}{|l|}{ Insônia } \\
\hline Sim & 32 & 22,5 \\
\hline Não & 108 & 76,1 \\
\hline \multicolumn{3}{|c|}{ Horas Dormidas à Noite } \\
\hline Menos de 7 horas & 115 & 81,0 \\
\hline Entre 7 e 9 horas & 25 & 17,6 \\
\hline \multicolumn{3}{|l|}{ Etilismo } \\
\hline Sim & 104 & 73,2 \\
\hline Não & 36 & 25,4 \\
\hline \multicolumn{3}{|l|}{ Tabagismo } \\
\hline Sim & 9 & 6,3 \\
\hline Não & 131 & 92,3 \\
\hline \multicolumn{3}{|c|}{ Usa Substâncias Alteram o Sono } \\
\hline Sim & 83 & 58,5 \\
\hline Não & 57 & 40,1 \\
\hline \multicolumn{3}{|c|}{ Satisfação Rendimento Acadêmico } \\
\hline Sim & 43 & 30,3 \\
\hline Não & 97 & 68,3 \\
\hline \multicolumn{3}{|c|}{ Pensou Abandonar o Curso } \\
\hline Sim & 64 & 45,1 \\
\hline Não & 76 & 53,5 \\
\hline
\end{tabular}

Na comparação dos aspectos sociodemográficos com os níveis de estresse percebido dos estudantes foi identificado maior escore de estresse percebido no sexo feminino ( $p=$ $0,0053)$, bem como nos alunos que referiram não realizar atividades extracurriculares $(p=0,0105)$ (Tabela 3 ).

Tabela 3. Comparação dos aspectos sociodemográficos com os níveis de estresse percebido dos 140 estudantes internos de um curso de medicina, Goiânia, Goiás, Brasil, 2020.

\begin{tabular}{|c|c|c|c|c|c|}
\hline \multirow{2}{*}{ Variáveis ( $N=140)$} & \multirow{2}{*}{$n$} & \multirow{2}{*}{$f(\%)$} & \multicolumn{3}{|c|}{ Estresse Percebido (PSS) } \\
\hline & & & Média & $D P$ & p-valor* \\
\hline \multicolumn{6}{|l|}{ Sexo } \\
\hline Masculino & 41 & 29,3 & 26,4 & 9,9 & \\
\hline Feminino & 99 & 70,7 & 30,8 & 7,6 & 0,0053 \\
\hline \multicolumn{6}{|l|}{ Idade (anos) } \\
\hline Até 24 anos & 95 & 67,9 & 29,2 & 9,2 & \\
\hline Acima de 24 anos & 45 & 32,1 & 30,1 & 7,1 & 0,5300 \\
\hline
\end{tabular}




\begin{tabular}{|c|c|c|c|c|c|}
\hline \multicolumn{6}{|l|}{ Procedência } \\
\hline Goiânia & 80 & 57,1 & 29,2 & 9,2 & \\
\hline Interior de Goiás & 36 & 25,7 & 30,8 & 9,0 & \\
\hline Outro Estado & 24 & 17,1 & 28,4 & 4,8 & $0,6684^{* *}$ \\
\hline \multicolumn{6}{|c|}{ Trabalha e Cursa Medicina } \\
\hline Sim & 52 & 37,1 & 29,5 & 8,5 & \\
\hline Não & 88 & 62,9 & 29,5 & 8,6 & 0,9916 \\
\hline \multicolumn{6}{|l|}{ Período da Medicina } \\
\hline Período 9 & 27 & 19,3 & 28,9 & 7,6 & \\
\hline Período 10 & 31 & 22,1 & 29,9 & 10,0 & \\
\hline Período 11 & 37 & 26,4 & 27,9 & 8,1 & \\
\hline Período 12 & 45 & 32,1 & 30,8 & 8,4 & 0,5256 \\
\hline \multicolumn{6}{|c|}{ Atividades Extracurriculares } \\
\hline Nenhuma & 9 & 6,4 & 34,9 & 8,9 & \\
\hline 1 Atividade & 86 & 61,4 & 29,6 & 7,8 & \\
\hline 2 Atividades & 38 & 27,1 & 29,4 & 7,8 & \\
\hline 3 ou mais Atividades & 7 & 5,0 & 20,7 & 14,5 & 0,0105 \\
\hline \multicolumn{6}{|l|}{ Estado Civil } \\
\hline Solteiro & 77 & 55,0 & 29,5 & 7,8 & \\
\hline Solteiro (Namorando) & 59 & 42,1 & 29,4 & 9,7 & \\
\hline Casado & 4 & 2,9 & 29,5 & 5,4 & 0,9991 \\
\hline \multicolumn{6}{|l|}{ Envolvimento Religioso } \\
\hline Forte & 44 & 31,4 & 29,8 & 8,0 & \\
\hline Fraco & 67 & 47,9 & 29,3 & 8,1 & \\
\hline Nenhum & 29 & 20,7 & 29,4 & 10,5 & 0,9670 \\
\hline \multicolumn{6}{|l|}{ Mora com Quem? } \\
\hline Pai, mãe ou irmãos & 97 & 69,3 & 29,6 & 8,9 & \\
\hline Sozinho & 37 & 26,4 & 29,4 & 8,2 & \\
\hline Parentes & 6 & 4,3 & 27,7 & 2,3 & $0,5502^{* *}$ \\
\hline
\end{tabular}

*Testes: 2 variáveis: teste $t$ de Student; 3 ou mais: ANOVA, $\left(^{* *}\right)$ Kruskal-Wallis

Em relação à comparação dos aspectos pessoais e clínicos com os níveis de estresse percebido, maiores escores de estresse percebido foram encontrados nos estudantes que referiram ter doença psiquiátrica diagnosticada $(p=0,0273)$, nos que estavam fazendo terapia psiquiátrica/psicológica ( $p$ $=0,0003)$, nos que referiram ter insônia $(p=0,0099)$, nos que dormiam menos de sete horas por noite $(p=0,0161)$, nos que afirmaram não estarem satisfeitos com o próprio rendimento acadêmico $(p<0,0001)$ e naqueles que já pensaram em abandonar o curso de medicina $(p=0,0016)$ (Tabela 4).
Tabela 4. Comparação dos aspectos pessoais e clínicos com os níveis de estresse percebido dos 140 estudantes internos de um curso de medicina, Goiânia, Goiás, Brasil, 2020.

\begin{tabular}{|c|c|c|c|c|c|}
\hline \multirow{2}{*}{ Variáveis ( $N=140)$} & \multirow{2}{*}{$n$} & \multirow{2}{*}{$f(\%)$} & \multicolumn{3}{|c|}{ Estresse Percebido (PSS) } \\
\hline & & & Média & $D P$ & p-valor* \\
\hline \multicolumn{6}{|c|}{ Frequência em Bares e Festas } \\
\hline Frequentemente & 49 & 35,0 & 28,1 & 9,4 & \\
\hline Às vezes & 65 & 46,4 & 29,7 & 7,7 & \\
\hline Raramente & 26 & 18,6 & 31,4 & 8,8 & 0,2695 \\
\hline \multicolumn{6}{|c|}{ Frequência Atividade Física } \\
\hline Frequentemente & 71 & 50,7 & 28,8 & 7,8 & \\
\hline Às vezes & 37 & 26,4 & 29,4 & 8,7 & \\
\hline Raramente & 32 & 22,9 & 30,9 & 9,9 & 0,5338 \\
\hline \multicolumn{6}{|l|}{ Doença Crônica } \\
\hline Sim & 12 & 8,6 & 31,0 & 5,7 & \\
\hline Não & 128 & 91,4 & 29,3 & 8,8 & 0,5188 \\
\hline \multicolumn{6}{|l|}{ Doença Psiquiátrica } \\
\hline Sim & 52 & 37,1 & 31,5 & 8,2 & \\
\hline Não & 88 & 62,9 & 28,3 & 8,6 & 0,0273 \\
\hline \multicolumn{6}{|c|}{ Terapia Psiquiátrica/Psicológica } \\
\hline Sim & 86 & 61,4 & 31,5 & 7,9 & \\
\hline Não & 54 & 38,6 & 26,3 & 8,6 & 0,0003 \\
\hline \multicolumn{6}{|l|}{ Insônia } \\
\hline Sim & 32 & 22,9 & 32,9 & 8,0 & \\
\hline Não & 108 & 77,1 & 28,5 & 8,5 & 0,0099 \\
\hline \multicolumn{6}{|c|}{ Horas Dormidas à Noite } \\
\hline Menos de 7 horas & 115 & 82,1 & 30,3 & 8,4 & \\
\hline Entre 7 e 9 horas & 25 & 17,9 & 25,8 & 8,6 & 0,0161 \\
\hline \multicolumn{6}{|l|}{ Etilismo } \\
\hline Sim & 104 & 74,3 & 29,5 & 8,9 & \\
\hline Não & 36 & 25,7 & 29,5 & 7,6 & 0,9815 \\
\hline \multicolumn{6}{|l|}{ Tabagismo } \\
\hline Sim & 9 & 6,4 & 25,6 & 7,7 & \\
\hline Não & 131 & 93,6 & 29,7 & 8,6 & 0,1560 \\
\hline \multicolumn{6}{|c|}{ Usa Substâncias Alteram o Sono } \\
\hline Sim & 83 & 59,3 & 29,9 & 8,9 & \\
\hline Não & 57 & 40,7 & 28,8 & 8,0 & 0,4358 \\
\hline \multicolumn{6}{|c|}{ Satisfação Rendimento Acadêmico } \\
\hline Sim & 43 & 30,7 & 23,1 & 8,5 & \\
\hline Não & 97 & 69,3 & 32,3 & 6,9 & $<0,0001$ \\
\hline \multicolumn{6}{|c|}{ Pensou Abandonar o Curso } \\
\hline Sim & 64 & 45,7 & 31,9 & 9,0 & \\
\hline Não & 76 & 54,3 & 27,4 & 7,6 & 0,0016 \\
\hline
\end{tabular}

*Testes: 2 variáveis: teste $t$ de Student; 3 ou mais: ANOVA, $\left(^{* *}\right)$ Kruskal-Wallis 


\section{Discussão}

Na literatura, o sexo feminino tem sido descrito como um fator de maior predisposição ao estresse e a desordens mentais. Isso tem sido associado com os múltiplos papéis exercidos pela mulher na sociedade atrelado as alterações de humor por mudanças hormonais. Assim, encontra-se associação estatisticamente significativa entre gênero e sintomas ansiosos, apresentando também mais chance de desenvolver depressão quando comparado aos homens, o que foi identificado em uma pesquisa que mostrou associação entre a quase exaustão dos estudantes de medicina e o sexo feminino ${ }^{8}$.

Corroborando os achados da presente pesquisa, cita-se um estudo realizado com 115 estudantes (61 homens e 54 mulheres) regularmente matriculados no curso de medicina no Sudoeste da Bahia, cujos resultados apresentaram prevalência de $32,2 \%$ de transtornos mentais comuns, sendo $70,3 \%$ desses, no sexo feminino. Ademais, em seu estudo, postulouse que estudantes de medicina do sexo feminino, quando comparadas aos do sexo masculino, possuem dificuldade em conciliar o internato com os estudos, ou seja, dificuldade com dedicação no período integral, especificamente no período do internato médico? .

Possíveis causas desse risco aumentado em mulheres são, além das interações entre influências hormonais, também as diferenças sexuais ligadas a aspectos neuronais envolvidas com humor e ansiedade, estressores psicossociais, papéis de gênero prescritos e a importância do apoio social para mulher ${ }^{10}$.

Outro aspecto relevante da presente pesquisa foi o maior escore de estresse percebido em quem não tinha nenhuma atividade extracurricular. A construção de um currículo paralelo, ou seja, a participação em ligas acadêmicas, iniciação científica, atividades artísticas e culturais, dentre outras, denota uma postura mais diretiva dos estudantes na própria formação, elementos que são essenciais para o processo ativo de aprendizagem. De simples receptores passivos de informações previamente preparadas pelo professor, os acadêmicos passam a ter posição ativa na construção do seu próprio conhecimento. Através da autonomia e participação ativa, os graduandos podem vivenciar o aprendizado com a construção e aplicação dos próprios pensamentos e ideias, o que coopera para a sua motivação com seu percurso acadêmico ${ }^{11}$.

Não participar das diversas atividades extracurriculares disponíveis pode significar que o estudante está trabalhando enquanto cursa medicina ou está dedicado de forma integral as atividades curriculares do curso. Corroborando com os dados da presente pesquisa, um estudo sobre fatores associados à Síndrome de Burnout em estudantes internos de medicina identificou que os alunos que participavam de atividades de iniciação científica tiveram melhor escore na dimensão satisfação pessoal e menor descrença ${ }^{12}$.

A entrada na universidade representa um momento de transição, e é por isso que o sentimento de adequação a determinado grupo e o apoio social podem auxiliar na adaptação dos alunos. Além disso, o aperfeiçoamento de habilidades, melhora das perspectivas acadêmicas e de mercado de trabalho e interação com colegas e profissionais, são benefícios advindos do desenvolvimento pessoal, social e intelectual dos estudantes. Através da formação de grupos e amizades fora do ambiente de sala de aula, o estudante encontra um suporte de apoio que se torna imprescindível para seu envolvimento na graduação, beneficiando assim, sua saúde física e mental por meio de um ambiente de distração paralelo e auxiliando no enfrentamento do estresse diário proporcionado pela graduação de Medicina. Nos raros tempos ociosos, os acadêmicos conseguem dedicar-se a atividades extracurriculares que não estão diretamente ligadas a graduação, como a prática de esportes e atividades culturais, que também influenciam diretamente na sua vida futura e trazem sensação de satisfação pessoal e proporcionam uma redução do estresse ${ }^{13}$.

No presente estudo, maiores níveis de estresse percebido foram encontrados nos estudantes que referiram ter doença psiquiátrica diagnosticada, bem como aqueles que faziam terapia psiquiátrica e/ou psicológica. Diversas características fazem com que o curso de Medicina seja poten $\neg$ cialmente atuante na qualidade de vida dos estudantes. Uma gama de fatores estressantes na educação médica pode levar a consequências drásticas na saúde desses alunos ${ }^{3}$.

Nesse sentido, o auxílio psicológico torna-se uma estratégia de enfrentamento, com diversos benefícios para os estudantes, haja vista que quem procura esse tipo de ajuda, geralmente se sente perdido, angustiado ou atormentado, sendo necessário o fornecimento de recursos frente às adversidades e dificuldades trazidas pelo curso, para se elaborar um modelo mais saudável de vida, melhor reestruturação e ressignificação do cotidiano estudantil ${ }^{14}$.

O processo de "tornar-se médico" causa sofrimento, e é um discurso fortemente reafirmado pela escola médica e pela sociedade, gerando uma naturalização do adoecimento psíquico dos estudantes de medicina e fazendo com que, comumente, os acadêmicos desenvolvam estratégias individuais de isolamento, negação, culpa, medo e o silêncio sobre o acometimento. Tudo isso, propicia o surgimento de um ciclo que estimula ainda mais o processo de desvalorização psíquica e exacerba as dificuldades de mudanças, cuidados e rupturas na vida do indivíduo ${ }^{15}$. 
O presente estudo demonstrou uma associação significativa entre estresse percebido e menores horas de sono e diagnóstico de insônia. Isso demonstra que dormir pouco, bem como a insônia são preditores para maiores níveis de estresse percebido. Fisiologicamente, sabe-se que o sono e o estresse estão intimamente ligados ao eixo hipotálamohipófise-adrenal (HPA), o que pode explicar a estreita relação entre esses dois fatores. O estresse agudo é acompanhado por uma diminuição da onda lenta e do movimento rápido dos olhos (REM), e a privação do sono, como estressor, tem efeitos pronunciados na arquitetura do sono e nos ritmos circadianos. Essas alterações características do eletroencefalograma do sono também ocorreram em pacientes com distúrbios afetivos, como depressão ${ }^{16}$.

Geralmente, devido às múltiplas demandas do ambiente acadêmico, os estudantes de medicina tendem a reduzir suas horas de sono para prolongar o tempo disponível para estudar. Eles podem não considerar o sono como a principal prioridade em comparação com os estudos e outros requisitos acadêmicos. Consequentemente, tornam-se privados de sono e estressados, especialmente nas semanas anteriores as provas. $\mathrm{O}$ resultado é um ciclo vicioso, no qual os alunos estão tentando lidar com seus múltiplos requisitos acadêmicos e estressores, reduzindo o tempo de sono, resultando em privação do sono e má qualidade do sono que aumentam seus níveis de estresse ${ }^{16}$.

Em um estudo realizado com 116 estudantes internos do curso de medicina em 2020, avaliou-se a associação entre a sonolência excessiva diurna e o desempenho acadêmico. Observou-se associação significativa entre os estudantes que relataram insatisfação com o rendimento acadêmico e que se sentiam extremamente sonolentos durante o dia. A redução do rendimento acadêmico, profissional e social é consequência dos prejuízos advindos da sonolência excessiva diurna, trazendo a curto e a longo prazo a redução das funções cognitivas (atenção, concentração, autoestima e humor), redução da vigilância, prejuízos na qualidade de vida, maiores chances de acidentes e o surgimento ou exacerbação de transtornos psiquiátricos ${ }^{17}$.

Os últimos anos de curso são considerados os mais estressantes para o estudante, principalmente devido à sobrecarga de tarefas que geralmente levam o formando a dedicar quase todo o seu tempo para a universidade em detrimento da sua vida social e familiar. Nessa fase o estudante precisa cumprir diversas atividades que lhes consomem muito tempo. Ademais, é importante salientar que na amostra estudada cerca de um terço estava trabalhando em concomitância com o curso. Além disso, há ainda as preocupações e as incertezas sobre a vida profissional e a entrada no mercado de trabalho cada vez mais competitivo ${ }^{18}$. Essas preocupações geram sentimentos de dúvida, insegurança, angústia, ansiedade e maiores níveis de estresse.

A saúde mental está relacionada com o rendimento acadêmico dos estudantes. No presente estudo foi possível observar que situações de estresse enfrentadas pelos alunos possuem um impacto negativo, gerando insatisfação com o rendimento acadêmico e pensamento de abandonar o curso. Com isso, tais aspectos se tornam preocupantes para a gestão dos cursos de medicina, uma vez que isso pode se tornar cada vez mais frequente e por isso, devem ser fatores merecedores de atenção, visando preservar a saúde mental dos estudantes, que serão os futuros médicos.

\section{Conclusão}

Os estudantes de medicina que participaram deste estudo representam uma população jovem, cujos resultados revelaram fatores sociodemográficos, pessoais e clínicos importantes associados a maiores níveis de estresse percebido no período do internato médico, são eles: ser do sexo feminino, não ter atividades extracurriculares, ter doença psiquiátrica diagnosticada, frequentar terapia psiquiátrica/psicológica, dormir menos de sete horas a noite, ter insônia, estar insatisfeito com o rendimento acadêmico e ter pensamento de abandonar o curso.

É importante observar, dentro do contexto, o quanto a rotina acadêmica pode influenciar diretamente sobre os níveis de estresse percebido entre os estudantes, reforçando novamente a importância de avaliar e preservar a saúde mental dos futuros médicos. A identificação dos grupos mais vulneráveis de forma precoce torna-se fundamental, a fim de uma intervenção efetiva visando à redução dos níveis de estresse no ambiente acadêmico. Embora o estudo seja restrito ao período do internato, é de grande relevância que novos estudos com essa temática sejam feitos, na perspectiva de melhorar a vida diária dos estudantes e ampliar o seu rendimento acadêmico. Assim, recomenda-se a realização de novas pesquisas em cenário mais abrangente e em diversas regiões do país. Espera-se que esse estudo forneça dados para execução de ações de promoção e prevenção relacionadas à saúde mental dos estudantes de medicina, principalmente aqueles que se encontram no internato médico.

\section{Referências}

1. Almojali Al, Almalki SA, Alothman AS, Masuadi EM, Alaqeel MK. The prevalence and association of stress with sleep quality among medical students. J Epidemiol Glob Health. 2017;7(3):169-74. 
2. Aragão J. Introdução aos estudos quantitativos utilizados em pesquisas científicas. Rev Práxis. 2017;3(6):59-62.

3. Conceição LDS, Batista CB, Dâmaso JGB, Pereira BS, Carniele RC, Pereira GS. Saúde mental dos estudantes de medicina brasileiros: uma revisão sistemática da literatura. Avaliação. 2019;24(3):785-802.

4. Costa LBS. Avaliação do estresse e do rendimento acadêmico em estudantes da área da saúde da Universidade de Brasília. Dissertação. Programa de PósGraduação em Ciências da Saúde. Universidade de Brasília. 89f. 2018.

5. Machado CS, Moura TM, Almeida RJ. Estudantes de medicina e as drogas: evidências de um grave problema. Rev Bras Educ Med. 2015;39(1):159-67.

6. Estrela YCA, Rezende ACC, Guedes AF, Pereira CO, Sousa MNA. Estresse e correlatos com características de saúde e sociodemográficas de estudantes de medicina. Rev CES Med. 2018;2(3):215-25.

7. Dias JCR, Silva WR, Maroco J, Campos JADB. Escala de estresse percebido aplicada a estudantes universitárias: estudo de validação. Psychology, Community \& Health. 2015;4(1):1-13.

8. Ferreira CMG, Kluthcovsky ACGC, Cordeiro TMG. Prevalência de transtornos mentais comuns e fatores associados em estudantes de medicina: um estudo comparativo. Rev Bras Educ Med. 2016;40(2):268-77.

9. Chagas MKS, Moreira Júnior DBM, Cunha GN, Caixeta RP, Fonseca EF. Ocorrência da síndrome de burnout em acadêmicos de medicina de instituição de ensino no interior de Minas Gerais. Rev Med e Saúde Brasília. 2016;5(2):234-45.

10. Lima RL, Soares MEC, Prado SN, Albuquerque GSC. Estresse do estudante de medicina e rendimento acadêmico. Rev Bras Educ Med. 2016;40(4):678-84.

11. Santos FS, Maia CRC, Faedo FC, Gomes GPC, Nunes ME, Oliveira MVM. Estresse em estudantes de cursos preparatórios e de graduação em medicina. Rev Bras Educ Med. 2017;41(2):194-200.

12. Moreira SNT, Vasconcellos RLSS, Heath N. Estresse na formação médica: como lidar com essa realidade? Rev Bras Educ Med. 2015;39(4):558-64.

13. Prado MSFM, Norte NM, Carvalho IGM, Sousa IF, Almeida RJ. Avaliação da Síndrome de Burnout entre estudantes do último ano de um curso de medicina do Brasil. Arq Ciências da Saúde. 2019;26(1):41-46.

14. Querido IA, Naghettini AV, Orsini MRCA, Bartholomeu $D$, Montiel JM. Fatores associados ao estresse no inter- nato médico. Rev Bras Educ Med. 2016;40(4):565-73.

15. Reis RS, Hino AA, Añez CR. Perceived stress scale: reliability and validity study in Brazil. J Health Psychol. 2010;15(1):107-14.

16. Santos LS, Ribeiro IJS, Boery EN, Boery RNSO. Qualidade de vida e transtornos mentais comuns em estudantes de medicina. Cogitare Enferm. 2017;22(4):4-10.

17. Vaz ALL, Gléria VO, Bastos GCFC, Sousa IF, Silva AMTC, Almeida RJ. Fatores associados aos níveis de fadiga e sonolência excessiva diurna em estudantes do internato de um curso de medicina. Rev Bras Educ Med. 2020;44(1):e011.

18. Souza AS, Tavares KM, Pinto PSP. Depressão em estudantes de medicina: uma revisão sistemática da literatura. Anais do XVI SEPA - Seminário Estudantil de Produção Acadêmica, UNIFACS. 2017;18:218-34. 